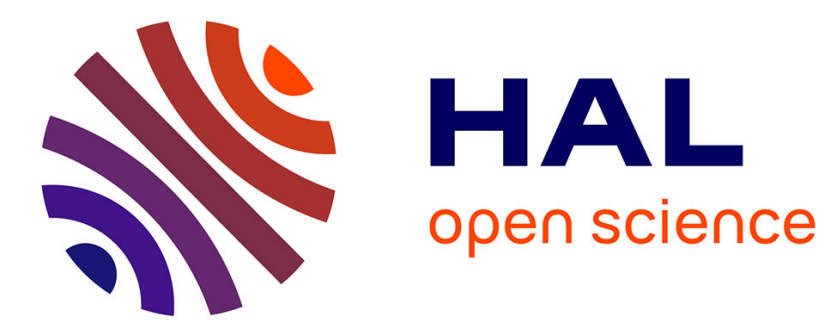

\title{
Les élections, principal théâtre de la crise de l'État égyptien
}

Clément Steuer

\section{To cite this version:}

Clément Steuer. Les élections, principal théâtre de la crise de l'État égyptien. Maghreb-Machrek, 2020, Les bouleversements politiques en Afrique du Nord au prisme de l'analyse électorale, $\mathrm{N}^{\circ} 243$ (1), pp.41-55. 10.3917/machr.243.0041 . hal-03128464

\section{HAL Id: hal-03128464 https://hal.science/hal-03128464}

Submitted on 2 Feb 2021

HAL is a multi-disciplinary open access archive for the deposit and dissemination of scientific research documents, whether they are published or not. The documents may come from teaching and research institutions in France or abroad, or from public or private research centers.
L'archive ouverte pluridisciplinaire HAL, est destinée au dépôt et à la diffusion de documents scientifiques de niveau recherche, publiés ou non, émanant des établissements d'enseignement et de recherche français ou étrangers, des laboratoires publics ou privés. 


\title{
Les élections, principal théâtre de la crise de l'État égyptien
}

\author{
Clément Steuer
}

\section{Biographie}

Clément Steuer est chercheur contractuel CNRS, UMR 7533 LADYSS et membre du projet TARICA de l'European Research Consortium. Il a publié en 2020 "Les Frères musulmans égyptiens face à la pluralisation de l'offre militante islamiste ", L'Année du Maghreb, $\mathrm{n}^{0}$ 22, p. 169-184

\section{Résumé}

Le système partisan représente un poste d’observation privilégié pour étudier la crise systémique de l'État égyptien. En même temps, il constitue une arène au sein de laquelle l'État cherche à résoudre cette crise à travers les élections, afin de trouver un nouveau modèle pour ses relations avec la société.

L’ancien parti hégémonique (le parti national démocratique, PND) était entré en crise dès les années 1990, du fait de la montée en puissance de nouvelles élites sociales, principalement les hommes d'affaires. Parallèlement, les partis d'opposition étaient trop faibles pour attirer ces nouvelles élites, ou même pour jouer un rôle dans l'expression de la demande sociale. Ils se sont également avérés incapables de remplir le vide laissé par la dissolution du PND en 2011, laissant le champ libre à l'islam politique, malgré la croissance du corps électoral et la politisation du vote pendant la période 2011-2013. Mais soutenus principalement par les zones pauvres et marginalisées du sud et des frontières du pays, les islamistes durent faire face à l'hostilité des populations du cœur de l'Égypte (Le Caire, Alexandrie, le Delta et le Canal) lorsqu’ils parvinrent au pouvoir en 2012.

Depuis leur chute en 2013, le régime a essayé de mettre en place différents modèles de lien entre l'État et la société : formation d'une nouvelle élite à travers la création du Parti du futur de la patrie, coalition nationale des partis séculiers existants, ou fusion desdits partis en trois ou quatre entités. Jusqu'à présent, toutes ces tentatives ont tourné court, et un nouveau modèle de relation entre l'État égyptien et sa société reste à trouver.

\section{Abstract}

The party system represents a privileged observation post for studying the systemic crisis of the Egyptian state. At the same time, it constitutes an arena in which the state seeks to resolve this crisis through elections, in order to find a new model for its relations with society.

The former hegemonic party (the National Democratic Party, NDP) had entered into crisis as early as the 1990s, due to the rise of new social elites, mainly businessmen. At the same time, the opposition parties were too weak to attract these new elites, or even to play a role in expressing social demand. They also proved unable to fill the vacuum left by the dissolution of the NDP in 2011, leaving the field open to political Islam, despite the growth of the electorate and the politicisation of the vote during the period 2011-2013. But supported mainly by the poor and marginalised areas in the south and along the country's borders, Islamists had to face hostility from the people in the heart of Egypt (Cairo, Alexandria, the Delta and the Canal) when they came to power in 2012.

Since their fall in 2013, the regime has tried to set up different models for linking the state and society: the formation of a new elite through the creation of the Future of the Homeland Party, a national coalition of existing secular parties, or the merging of the aforesaid parties into three or four entities. So far, all these attempts have failed, and a new model for the relationship between the Egyptian state and its society has yet to be found. 
S’il est un domaine dans lequel la révolution égyptienne de 2011 a entraîné des effets spectaculaires et quasi-immédiats, c'est celui du champ partisan, qui a connu en quelques années deux restructurations majeures, passant d'un système hégémonique à un système bipolaire, puis fragmenté ${ }^{1}$. Cette instabilité du champ partisan s'explique en grande partie par la faiblesse des partis, qui constituent le maillon le plus fragile du système politique égyptien, incapables qu'ils sont d'exercer leurs fonctions de sélection des élites, de mobilisation des électeurs, d'agrégation des intérêts et de transmission de la demande sociale auprès du pouvoir central. Il est donc compréhensible que ce sous-système particulièrement sensible soit le plus affecté par la crise systémique globale de l'État égyptien, elle-même emblématique de la crise des États arabes, dont le « Printemps » de 2010-2011 a été le révélateur.

Ces deux restructurations brutales (induites respectivement par la révolution de 2011, puis par la reprise en main autoritaire de 2013) ayant affecté le champ partisan égyptien au cours des dernières années ont été accompagnées par des réformes de l'environnement juridique dans lequel opèrent les partis politiques, mais aussi et surtout par d'importantes mutations du corps électoral. Alors que sous Moubarak (1981-2011), ce dernier était numériquement faible, pauvre et rural, il voit sa taille multipliée par quatre pour la durée des élections de 2011-2012 (durant la transition conduite par le Conseil supérieur des forces armées, puis sous la présidence de Muhammad Morsi), gonflé par une population urbaine et diplômée ayant enfin trouvé le chemin des urnes dans le contexte d'ouverture politique consécutif à la révolution ${ }^{2}$. Si la structure de l'électorat d'ancien régime était caractéristique d'un vote clientéliste, celui de la période révolutionnaire tendait ainsi à se rapprocher du modèle idéal-typique du marché politique. En ce domaine comme en beaucoup d'autres, la restauration autoritaire entamée en juillet 2013 (sous la présidence intérimaire de Adly Mansour, puis sous la présidence de Abdelfattah al-Sissi) n'a pas conduit à un retour au statu quo ante, mais plutôt à ce qui ressemble à une esquisse quelque peu scolaire de dépassement hégélien : l'électorat égyptien post-2013 est toujours deux fois plus étendu que celui d'ancien régime, même s'il ne représente plus que la moitié de l'électorat de la période révolutionnaire (du fait surtout d'un recul spectaculaire de la participation de la jeunesse). Bien que tendant à se dépolitiser - dans un contexte faisant de l'alternance un horizon plus que jamais hors de portée - cet électorat ne peut plus être mobilisé par le seul recours aux réseaux clientélistes traditionnels, et le régime se trouve désormais contraint d'employer une palette de plus en plus large d'expédients lui permettant de maintenir un taux de participation respectable.

Cet article entend analyser les transformations récentes du sous-système partisan égyptien, à partir du postulat selon lequel ces transformations expriment une crise majeure de l'État lui-même, et de ses rapports avec la société. Cette crise a d'abord connu une longue période de gestation, qui a vu les tensions s'aggraver au sein du parti national démocratique (PND) dès la deuxième moitié des années 1990, ${ }^{3}$ avant d'éclater au grand jour lors de la révolution de janvier 2011, qui se solda par la dissolution dudit parti hégémonique. Or, celui-ci remplissait dans le système moubarakien une fonction d'organisation des réseaux de clientèle qui liaient le centre du pouvoir étatique à la société égyptienne par des rapports clients/patrons en cascade. Depuis lors, toutes les tentatives de remplacer le défunt parti présidentiel - par le jeu normal de la concurrence électorale, par

1 Sur ces notions, voir Giovanni Sartori, Parties and Party Systems. A Framework for Analysis, Cambridge, Cambridge University Press, 1976.

2 Mahmoud Abou Kassem, Mona Amer, Akram El-Alphy et Sarah Ben Néfissa, « Dépolitisation de l’urne et politisation de l'électeur en Égypte : quelques enseignements des élections législatives égyptiennes 2015 », Confluences Méditerranée, n 102, 2017, p. 181-196.

3 Lisa Blaydes et Safinaz El Tarouty, « La concurrence interne au Parti national démocrate égyptien », Égypte/Monde arabe, $3^{\mathrm{e}}$ série, $\mathrm{n}^{\circ} 7,2010$, p. 69-93. 
l'hégémonie d'une contre-élite islamiste incarnée par l'organisation des Frères musulmans, ou par la tutelle de l'appareil sécuritaire sur le système partisan - ont échoué. Dix ans après la révolution, le lien entre la société et l'État n'a toujours pas été rétabli, et le projet d'une fusion plus ou moins volontaire des nombreuses organisations partisanes égyptiennes en trois ou quatre grands partis, semble avoir fait long feu.

Les opérations électorales constituent ainsi à la fois le mode d'expression le plus lisible de la crise de l'État égyptien, et le principal laboratoire de recherche d'une solution à cette crise. Le terme de « théâtre » employé dans le titre de cet article doit donc être compris dans les deux sens du terme : mise en scène de la tragédie en cours (parfois agrémentée, il est vrai, d'éléments propres à la comédie du pouvoir) et théâtre d'opération privilégié, où s'affrontent différents modèles de résolution de la crise et de rétablissement d'une relation stable entre l'État et la société.

Après avoir rappelé le lien désormais bien établi entre renouvellement des élites, faiblesse des partis politiques et crise de l'État égyptien, nous nous pencherons sur les mutations du corps électoral et des pratiques de vote, avant de montrer ce que les restructurations récentes du système partisan peuvent nous apprendre sur la société égyptienne et son rapport à l’État.

\section{Le renouvellement des élites, la faiblesse des partis et la crise de l’État}

Le système partisan prévalant durant les trente années de règne de Hosni Moubarak peut être défini, en reprenant la taxinomie de Sartori ${ }^{4}$, de système à parti hégémonique, à savoir un système dans lequel certains partis politiques sont autorisés à participer à la compétition électorale, aussi longtemps qu'ils ne constituent pas une menace pour la perpétuation de la domination du parti au pouvoir. Pour maintenir cette domination, ledit parti n’hésite pas à recourir le cas échéant à des moyens légaux (interdiction de tel ou tel parti représentant une menace potentielle) aussi bien qu'illégaux (violence électorale ${ }^{5}$, fraudes). Depuis sa création en 1978, le PND a en effet systématiquement occupé plus de deux tiers des sièges au Parlement (soit la majorité qualifiée), mais jamais leur totalité, laissant toujours quelques députés à au moins un parti de l’opposition.

À l’origine, le PND a été conçu comme l’héritier du parti unique de la période nassérienne (l’Union socialiste arabe) ou tout au moins de sa tendance « centriste ", largement majoritaire. Les élections législatives de 1976 virent en effet s'affronter pour la première fois, à l'initiative du président Anouar al-Sadate, trois tendances (ou « tribunes ») du parti unique. Sur 350 sièges alors pourvus au scrutin binominal majoritaire à deux tours, 295 furent remportés par l'organisation arabe socialiste (centriste), 15 par les libéraux (aile droite) et 3 par les unionistes (aile gauche). Suite à l'introduction du multipartisme par la loi $n^{\circ} 40$ de 1977, l'aile gauche du PND donna naissance au parti du Rassemblement (hizb al-tagammu') et son aile droite au parti des Libéraux (hizb al-ahrâr) ${ }^{6}$. Le 9 juillet 1978, le président Sadate annonça la création du PND, auquel se rallièrent presque instantanément 250 des 295 députés de l’organisation arabe socialiste. Des élections se tinrent en 1979, et le PND occupa 347 des 392 sièges que comptait alors l’Assemblée.

Du fait de ces origines monopartites, le PND occupa dans un premier temps l'une des fonctions caractéristiques de tout parti unique, à savoir celle d'un instrument de sélection et de cooptation des élites locales aux mains de la haute administration. Par contre, il s'est toujours distingué d'un parti unique en ceci qu'il ne remplissait guère de fonction de mobilisation de la population au service du projet politique porté par le régime. Les partis hégémoniques n’accordent en effet pas une place centrale à l'idéologie, et représentent même l'un des principaux outils de dépolitisation de la société aux mains des gouvernants. Dans le cas du PND, cette dépolitisation s'explique en grande partie par

4 Ibid., p. 230-231.

5 Violences directes ou intimidations visant les équipes, voire les électeurs présumés, des opposants. Au cours des années 2000, cette violence a été dans une grande mesure « privatisée », les candidats du PND embauchant des hommes de main pour effectuer les basses œuvres. Voir Iman Farag, "Corrompre, fidéliser : Les ressorts "légitimes” de la compétition électorale égyptienne », Égypte/Monde arabe, $3^{\mathrm{e}}$ série, nº 7, 2010, p. 21-46.

6 Si ces deux partis existent toujours à l'heure actuelle, seul le premier a été en mesure de s'assurer une présence constante au Parlement, alors que le second ne dispose plus d'aucun élu depuis 1990. 
les circonstances de sa fondation ${ }^{7}$, à une époque où Sadate avait définitivement rompu avec les orientations socialistes et pro-soviétiques de son prédécesseur, sans pour autant assumer pleinement son ralliement à l'économie de marché et au bloc occidental. Il en a résulté une absence d'épine dorsale idéologique pour son régime comme pour son parti, absence dont s'est fort bien accommodé son successeur, Hosni Moubarak. Sous la présidence de ce dernier (1981-2011), les principes socialistes demeurèrent gravés dans le marbre constitutionnel, alors même que les acquis sociaux et les grands groupes nationaux hérités de la période nassérienne (comme l'entreprise de textile Shebin, la chaîne de magasins Omar Effendi, ou encore l'Arab Company For Trade, et la Tanta Flax and Oils Company) étaient progressivement démantelés et privatisés. L'absence d'accountability du régime lui permettait d'étaler dans le temps ces réformes d'inspiration libérale, sans jamais les assumer pleinement, afin d'en atténuer les conséquences fâcheuses pour le pouvoir

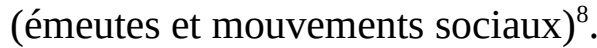

Mais même la fonction de sélection des élites occupée par le PND se transforma au cours du temps, ce parti devenant progressivement une structure lâche, organisant la compétition des élites au niveau local et donnant son onction aux vainqueurs bien davantage qu'il ne les choisissait. Ce phénomène est directement observable - de 1990 à 2005 - à travers l'augmentation du nombre de candidats «indépendants » élus lors des élections législatives. Dès 1990, en effet, 83 députés indépendants (sur 444) furent élus à l'Assemblée du peuple, dont plusieurs rejoignirent le parti hégémonique après l'élection. Pour beaucoup, il s'agissait de candidats qui avaient échoué à obtenir l'investiture du PND, mais se présentaient néanmoins contre le candidat officiel du parti (les «indépendants sur les principes du PND»). Cela leur permettait de tenter leur chance malgré l'absence de soutien du parti hégémonique, sans attaquer ce dernier de front. Du côté du pouvoir et de dirigeants du parti, cette situation leur permettait de sélectionner les hommes les plus forts de chaque circonscription (remporter les élections nécessitant d'importantes ressources) en utilisant les élections comme une arène de compétition entre les différents soutiens locaux du régime. En 1995, les candidats indépendants furent au nombre de 112 à être élus, dont 99 se rallièrent sans attendre au PND. En 2000, pour la première fois depuis sa création, le parti au pouvoir échoua à faire élire une majorité de ses candidats officiels (seulement 175 sur 444, soit $39 \%$ ). Il parvint néanmoins une nouvelle fois à obtenir une majorité qualifiée à la Chambre, car 178 de ses membres furent élus en tant qu' "indépendants sur les principes du PND ». Par ailleurs, 35 parmi les 72 indépendants restants joignirent le PND une fois élus. Enfin, en 2005, seuls 151 candidats officiellement investis par le PND furent élus (34 \%), ce qui n’empêcha pas ce dernier d'occuper au final 73 \% des sièges (330 députés) grâce au ralliement de la majorité des indépendants.

Cette multiplication des candidats indépendants sur les principes du PND doit être mise en relation avec la montée en puissance concomitante des hommes d'affaires ${ }^{9}$, « signe tangible de la privatisation du clientélisme électoral égyptien ${ }^{10}$ ». Le système mis en place à la fin de l'ère sadatienne reposait en effet sur une alliance entre une partie des bureaucrates issus de l'ère nassérienne (actifs dans l'appareil d'État aussi bien que dans le PND et dans les syndicats) et les notabilités locales traditionnelles (chefs de tribus et de grandes familles, leaders religieux). Cette

7 De ce fait, ces circonstances expliquent sans doute pourquoi le besoin s'est alors fait sentir pour le régime de rompre avec le monopartisme et d'autoriser un multipartisme limité, passant ainsi d'un système à parti unique à un système à parti hégémonique.

8 Jean-Noël Ferrié parle à ce propos de «statu quo évolutif » («Les ressorts de l'équilibre politique durant la présidence de Hosni Moubarak », dans Vincent Battesti et François Ireton, L’Égypte au présent, Paris, Sindbad/Actes Sud, 2011, p. 323-341, p. 324). Voir aussi, du même auteur, L'Égypte entre démocratie et islamisme. Le système Moubarak à l'heure de la succession, Paris, Autrement, 2008, et « Dispositifs autoritaires et changements politiques. Les cas de l’Égypte et du Maroc », Revue internationale de politique comparée, vol. 19, n 4, 2012, p. 93-110.

9 Voir Sarah Ben Néfissa et Alâ' Al-dîn Arafat, Vote et démocratie dans l'Égypte contemporaine, Paris, Khartala-IRD, 2005.

10 Marie Vannetzel, “"Ils nous ont déjà essayés !” Clientélisme et mobilisation électorale frériste en Égypte », Politique africaine, $\mathrm{n}^{\circ}$ 108, 2007, p. 47-66. Sur les différents types de pratiques clientélistes et leurs mutations, voir aussi Tewfik Aclimandos, «Splendeurs et misères du clientélisme », Égypte/Monde arabe, $3^{\mathrm{e}}$ série, n 7, 2010, p. 197219. 
alliance s'était faite principalement au détriment des classes moyennes diplômées (médecins, pharmaciens, ingénieurs, avocats), grandes perdantes des années 1970 sur tous les plans (économique, social, politique) et qui commencèrent dès cette époque à rallier en masse la contestation islamiste. Quant aux ouvriers et paysans, qui s’étaient déjà vus confisquer une grande partie de leur agency politique sous le régime des Officiers libres, leurs acquis sociaux et symboliques ne furent que très progressivement remis en cause, et le développement de mouvements contestataires en leur sein ne se fit pas sentir avant le milieu des années 1990, avec une intensité alors encore très faible.

La montée en puissance politique des hommes d'affaires, visible dès le milieu des années 1990 doit de son côté être interprétée comme un effet différé de la politique d'ouverture économique (infitâh) décidée par Sadate en 1973. Elle se traduisit d'une part par l'intensification de la compétition électorale au niveau local, et d'autre part par une division des élites au niveau national. Les milieux d'affaires trouvèrent en effet rapidement des alliés au sein du régime, en la personne d'universitaires modernisateurs et d'une couche montante de technocrates formés en Occident. Le PND devint rapidement le principal champ de bataille opposant une "vieille garde » dont les figures emblématiques étaient des serviteurs de l'État de longue date, tels que Kamâl al-Shazlî, Safwat al-Sharîf, ou encore Mufîd Shahâb, à une "jeune garde » incarnée par un groupe de quadragénaires proches des milieux d'affaires, dont les leaders n'étaient autres que le fils puîné du président de la République, Gamâl Moubarak, flanqué du magnat de l'acier Ahmad `Ezz ${ }^{11}$.

De leur côté, les partis politiques d'opposition - bien qu'autorisés sous condition depuis 1977 s'avérèrent incapables d'attirer autrement qu'à la marge l'élite montante des hommes d'affaires ainsi que la contre-élite contestataire des travailleurs en col blanc. Cette "faiblesse ${ }^{12}$ des organisations partisanes égyptiennes s'explique à la fois par l'appareillage légal mis en place par le régime pour limiter la compétition (voir infra), et par leur insertion dans le jeu clientéliste dès le début des années $1990^{13}$. De ce fait, les partis d'opposition présents au Parlement recrutaient leurs cadres prioritairement parmi des catégories sociales en déclin depuis la fin de l'ère nassérienne (la bureaucratie, notamment ouvrière, au sein du parti du Rassemblement, socialiste, et du parti arabe démocratique nassérien), voire de la monarchie constitutionnelle (les anciens grands propriétaires terriens au sein du libéral Parti Wafd). Preuve des dysfonctionnements du système, seuls les partis officiellement illégaux (le néo-nassérien Parti Karâma, le Parti Wasat, islamiste modéré, et surtout les Frères musulmans) parvenaient à attirer de manière significative des représentants des classes moyennes diplômées et des hommes d'affaires contestataires, entraînant une vitalité du « champ politique informel $\gg{ }^{14}$ contrastant avec l'atonie de l'opposition formelle.

La faiblesse des partis d’opposition explique également leur quasi-incapacité à se faire les relais de la demande sociale auprès du pouvoir central, fonction qu'ils laissent alors volontiers au PND, comme l'illustre notamment la manière dont a été géré en 2008 le mouvement social né à Damiette, en réaction à l'annonce de la construction d'une usine d'engrais chimique particulièrement polluante dans cette ville côtière ${ }^{15}$. La mobilisation populaire locale contre ce projet s'était faite dans un premier temps à l'initiative des mouvements et partis politiques d'opposition, aussi bien légaux (Wafd, parti du Rassemblement, parti arabe démocratique nassérien) qu’illégaux (Frères musulmans, Wasat). Si ces partis politiques parvinrent à pousser les classes moyennes diplômées à entrer dans la lutte, ils manquaient de relais au sein du milieu ouvrier, et surtout s'avérèrent

11 Voir Lisa Blaydes et Safinaz El Tarouty, op. cit.

12 Sarah Ben Néfissa, «Les partis politiques égyptiens entre les contraintes du système politique et le renouvellement des élites ", Revue des mondes musulmans et de la Méditerranée, vol. 81, n 1, p. 55-91.

13 May Kassem, In the Guise of Democracy: Governance in Contemporary Egypt, Reading, Ithaca Press, 1999.

14 Alain Roussillon, «Les nouveaux partis politiques, note sur le processus de recomposition du système politique égyptien ", Égypte/Monde arabe, $1^{\text {ère }}$ série, n ${ }^{\circ}$ 2, 1990, p. 123-142 ; Sarah Ben Néfissa, op. cit. Sur la postérité de cette notion, voir aussi Clément Steuer, "Alain Roussillon et le renouveau de l'étude des partis politiques égyptiens ", Égypte/Monde arabe, $3^{\text {e }}$ série, $\mathrm{n}^{\circ}$ 20, 2019, p. 111-125.

15 Clément Steuer, « Les causes environnementales en Égypte : de l'échec de la politisation par le haut au succès des mobilisations par le bas », Mouvements, n 80, 2014, p. 24-33. 
incapables d'exercer la moindre influence sur le pouvoir. De leur côté, les hommes d'affaires locaux - dont les intérêts économiques étaient directement menacés par le projet de construction d'une industrie polluante - firent entendre leur voix par l'intermédiaire de la chambre de commerce de Damiette, avant de rallier à leur cause les conseils populaires municipaux des villes environnantes, ainsi que le conseil populaire du gouvernorat, toutes instances dominées par le PND. La section locale de ce dernier fit alors elle aussi part de son opposition au projet, entraînant avec elle les élus des deux chambres, puis le gouverneur. Durant l'été 2008, l'Assemblée du peuple créa une commission d'enquête sur cette affaire - commission d'ailleurs dominée par des députés PND, mais comportant également des membres de l'opposition -, avant de voter unanimement en faveur d'une relocalisation du projet. Au mois d'août, le gouvernement dénonça l'accord d'investissement qui le liait à l'entreprise canadienne en charge de la construction de l'usine.

L'on voit ainsi que la capacité du PND à jouer un rôle de relais de la demande sociale reposait à la fois sur le développement d'une certaine agency de ses élus au Parlement, désormais beaucoup moins dépendants de l'investiture accordée par leur parti que de leur aptitude personnelle à mobiliser des électeurs en leur faveur, et sur le maintien de son hégémonie au niveau local. Ce maintien passait en grande partie par sa capacité - alors toujours intacte - à sélectionner les élus au sein des conseils populaires locaux. En effet, si ces derniers ne disposaient que d'un pouvoir strictement consultatif dans la gestion des affaires locales, ils n'en constituaient pas moins une porte d'accès aux ressources de l'État au niveau local, et un tremplin pour une éventuelle carrière politique $^{16}$. Or, lors des dernières élections locales organisées en Égypte, en avril 2008, le PND obtint sans difficultés $98 \%$ des sièges.

À la fin des années 2000, le système politique égyptien semblait ainsi robuste malgré la faiblesse des partis d'opposition. Le PND avait en effet su s'adapter pour intégrer une partie des élites montantes et rééquilibrer quelque peu le rapport de force entre l'État et la société au profit de cette dernière. De ce fait, il s'était montré capable de se faire à l'occasion le relais des demandes sociales auprès de l'État. Ce système s'avérait cependant incapable de répartir de manière équitable les ressources de l'État, les régions rurales (surtout dans le Sud) et les quartiers informels des grandes villes ayant été quasiment abandonnés par l'administration et laissés au bon soin des organisations caritatives - que celles-ci soient privées, aux mains des hommes d'affaires, ou religieuses, aux mains de l'Église, des Frères musulmans ou de la Prédication salafiste ${ }^{17}$ - ce qui aura des conséquences importantes lors des élections suivant la révolution de 2011. Surtout, comme nous le verrons dans la dernière partie de cet article, la division des élites entre « jeune garde » et " vieille garde » au sein du PND a servi d'élément déclencheur à la crise révolutionnaire, et a précipité son dénouement.

\section{Les mutations du corps électoral et la redéfinition de la signification du vote}

La révolution de 2011 a entraîné une mutation du corps électoral, tant en quantité (augmentation du nombre de votants potentiels) qu'en qualité (modification du ratio votants/inscrits, et de la composition sociale du corps des votants). Cette mutation s'est accompagnée d'une modification de la signification à accorder à l'acte électoral ${ }^{18}$.

Sous Moubarak, en effet, le corps électoral était explicitement limité aux citoyens en âge de voter inscrits sur les listes électorales ${ }^{19}$, ces dernières étant gérées par le ministère de l'Intérieur. Juridiquement, ce corps électoral se composait alors de deux strates : les citoyens nés avant 1982 ayant fait explicitement une demande d'inscription, et les citoyens nés à partir de cette date, théoriquement inscrits d'office. Dans les faits, ces listes comportent de nombreuses imperfections : électeurs non-inscrits en dépit de l'automaticité théorique ; double voire triple inscription d'un

16 Sarah Ben Néfissa, « Les assemblées d’arbitrage en Égypte », Égypte/Monde arabe, $3^{e}$ série, n ${ }^{\circ}$ 1, 2005, p. 55-72. 17 Sarah Ben Néfissa, Pouvoirs et associations dans le monde arabe, Paris, CNRS Édition, 2002 ; Marie Vannetzel, op. cit. Voir aussi, du même auteur, Les Frères musulmans égyptiens : enquête sur un secret public, Paris, Karthala, 2016. 18 Mahmoud Abou Kassem, et al., op. cit. 19 Sur cette question, voir Iman Farag, op. cit. 
même individu ; maintien d'électeurs décédés ou expatriés (les expatriés n’avaient alors pas le droit de vote). En 2005, il y avait 32 millions de personnes inscrites sur ces listes, soit seulement deux tiers des Égyptiens en âge de voter. Avec un taux de participation de $26 \%$, ces élections n'avaient d'ailleurs mobilisé que 8 millions d'électeurs. Au lendemain de la chute de Moubarak, afin de rompre avec les pratiques de fraudes associées à l'ancien régime, le Conseil supérieur des forces armées (CSFA) alors au pouvoir décide de supprimer ces listes. Désormais, tout citoyen de plus de 18 ans peut voter sur simple présentation de sa carte d'identité. Le corps électoral est ainsi passé brusquement à 45 millions de votants potentiels ${ }^{20}$, et a depuis lors continué à augmenter à un rythme soutenu, du fait de la croissance démographique de la population (53,6 millions en mai 2014, 59,1 millions en mars 2018). En conséquence, dès le 19 mars 2011 (référendum constitutionnel), plus de 18,5 millions d'électeurs se rendent aux urnes, soit plus du double des électeurs habituellement mobilisés sous Moubarak.

En effet, non seulement le corps électoral a-t-il rapidement augmenté en volume, mais le taux de participation a lui aussi cru au cours de la période révolutionnaire. Ainsi, lors des premières élections présidentielles organisées sous le règne de Hosni Moubarak, le taux de participation fut seulement de 22,9\% (7,1 millions d'électeurs), contre $46 \%$ au premier tour des élections de 2012 et $52 \%$ au second tour (25,6 millions d'électeurs). Le taux de participation déclina lors des élections présidentielles suivantes, mais demeura relativement élevé : 47,5 \% en 2014 (25,3 millions d'électeurs) et 41,5 \% en 2018 (24,3 millions d'électeurs). Concernant les élections parlementaires, on peut observer un phénomène similaire, quoique avec un déclin nettement plus prononcé dans la phase post-2013 ${ }^{21}$ : $26 \%$ en 2005, $54 \%$ en 2011-2012 et 28,3\% en 2015 (néanmoins, en chiffres absolus, et du fait de l'accroissement du corps électoral, ce dernier résultat représente toujours deux fois plus d'électeurs que ce que l'on était accoutumé à voir sous l'ancien régime).

Ces fluctuations du volume de l'électorat reflètent de profondes modifications de sa composition sociale. Ainsi, durant les deux dernières décennies du règne de Moubarak, les électeurs se recrutaient principalement « là où subsist[ai]ent encore des institutions sociales plus ou moins soumises au contrôle par consensus : couches aisées de la paysannerie et catégories ouvrières ${ }^{22}$. Durant la phase révolutionnaire, en 2011-2012, l'électorat urbain - et en premier lieu les classes moyennes diplômées - étaient venues grossir les chiffres de la participation électorale. Après la reprise en main du pays par l'armée en 2013 et avec le reflux de la mobilisation révolutionnaire, la jeunesse égyptienne a commencé à se détourner des urnes, laissant le vote aux classes moyennes supérieures éduquées et âgées, principalement en milieu rural ${ }^{23}$.

Ces mutations du corps électoral sont dues principalement ${ }^{24}$ aux différentes réformes du cadre juridique réglementant les élections et la vie partisane. Ainsi, comme vu supra, l'acte de vote s'est transformé au cours des trois décennies du règne de Moubarak : conçues à l'origine par le président Sadate comme un instrument voué à conférer l'onction populaire aux candidats choisis par l'administration, les élections sont progressivement devenues la sanction d'un rapport de force interne à la circonscription, un moyen de sélectionner par la compétition les élites locales ayant accumulé le plus de puissance sociale. Dès lors, les députés n'étaient plus seulement des représentants du pouvoir central, mais également ceux des groupes sociaux qui leur avaient permis de remporter l'élection ${ }^{25}$. En outre, le recul constant des candidats officiels du PND au profit des

20 D’autant que les expatriés s'étaient vu dans le même temps reconnaître le droit de vote.

21 Le recours à la moyenne est ici rendu nécessaire par le fait que ces élections ont toutes été disputées en deux ou trois phases de deux tours chacun.

22 Al-Ahrâm, 14 novembre 2005, cité dans Iman Farag, op. cit.

23 Mahmoud Abou Kassem, et. al., op. cit.

24 L'évolution démographique joue également un rôle majeur dans ces évolutions, mais elle joue ici le rôle d’une variable externe au système politique, du fait du faible degré de contrôle que ce dernier exerce sur elle, et de sa temporalité bien plus lente.

25 Élisabeth Longuenesse, «Les élections de novembre 1995. Le cas de la circonscription 25 à Hélouan », dans Sandrine Gamblin (dir.), Contours et détours du politique en Égypte. Les élections de 1995, Paris, L’Harmattan/CEDEJ, 1997, p. 229-266. 
indépendants - décrit supra - a pu être interprété comme « un vote prudent de sanction ${ }^{26}$, témoignant des prémisses d'une politisation du corps électoral.

Or, si la multiplication des candidatures indépendantes reflète la montée en puissance de nouvelles élites issues du monde économique, leur entrée en politique et leur capacité à se faire élire ont été rendues possibles par une importante modification du cadre juridique : le passage du scrutin proportionnel au scrutin individuel. Les élections législatives de 1984 et 1987 avaient été organisés au scrutin de $\operatorname{liste}^{27}$, avec principalement deux effets : l'administration était libre de choisir les candidats du PND qu'elle incluait sur ses listes, et les partis d'opposition légaux étaient tentés d'ouvrir leurs listes aux candidats des Frères musulmans afin d'attirer leurs électeurs. En 1987, puis en 1990, deux arrêts de la Haute cour constitutionnelle (HCC) déclarèrent illégales les lois électorales ayant présidé à l'organisation de ces scrutins, entraînant par deux fois une dissolution de l'Assemblée du peuple. La HCC considérait en effet que le scrutin de liste contrevenait au principe constitutionnel d'égalité des citoyens égyptiens, puisqu'elle favorisait les membres des partis politiques au détriment des indépendants.

C'est pour respecter cette jurisprudence constitutionnelle que toutes les élections législatives organisées entre 1990 et 2010 le furent intégralement au scrutin individuel. En autorisant les indépendants à se présenter, cette modification du cadre juridique rendit possible la compétition au sein de chaque circonscription de plusieurs candidats se réclamant du PND. Elle permit également aux Frères musulmans de se passer des services des partis politiques légalement reconnus, puisqu'il leur était désormais possible de présenter des candidats sous l'étiquette "indépendants ». Cette réforme eut donc pour effet de bord une aggravation de la faiblesse des partis d'opposition, un accroissement de leur dépendance au bon vouloir du régime, et au final leur cooptation au sein du jeu clientéliste qui se mettait alors en place. Alors que les avantages conférés par la légalité les enchaînaient déjà au pouvoir central, ils devaient désormais en outre se ménager les bonnes grâces du PND pour pouvoir survivre au sein du Parlement. En effet, privés du renfort des voix mobilisées par les Frères musulmans, leurs candidats n'avaient souvent d'autres choix pour être élus que de partager certaines circonscriptions avec le parti au pouvoir (en ne présentant pas de candidat sur le siège réservé aux ouvriers et paysans, en échange d'une absence d'investiture du PND pour le siège reservé aux autres catégories professionnelles dans la même circonscription. Ou inversement). Par ailleurs, dix membres de l'Assemblée du peuple (et un tiers de l'Assemblée consultative) étant nommés par le président de la République, celui-ci pouvait s'en servir pour offrir une présence au Parlement à tel ou tel parti « d'opposition $»^{28}$.

En 2011, l'augmentation spectaculaire du corps électoral est due également pour partie à la modification du cadre juridique : outre la suppression des listes électorales évoquée supra, cette période est en effet marquée par une réforme de la loi des partis (30 mars 2011) et par la dissolution du PND (16 avril 2011). L'effet le plus important de ladite réforme fut de casser le verrou que la loi de 1977 avait placé à l'entrée du champ politique, en instituant une commission des partis disposant d'un droit de vie et de mort sur les partis politiques, et dominée dans sa composition par les fidèles du chef de l'État, qui était en même temps président du PND. En stipulant que la majorité des membres de cette commission serait désormais composée de juges indépendants, cette réforme mit fin au contrôle du pouvoir sur l'offre politique, avec comme conséquence une multiplication des partis politiques, non seulement islamistes - qui venaient combler un vide - mais également socialistes et libéraux, qui disputèrent aux partis anciennement cooptés leurs monopoles sur différents segments du marché électoral : le Wafd dut ainsi faire face à la concurrence du Parti des Égyptiens libres et du Parti égyptien social-démocrate, tandis que le Parti du Rassemblement se voyait dépasser par le Parti de l'Alliance populaire socialiste. Ce soudain élargissement de l'offre politique fut d'autant plus spectaculaire que le parti hégémonique avait entre-temps été dissous par

26 Sarah Ben Néfissa et Alâ’ Al-dîn Arafat, op. cit., p. 286.

27 Intégralement en 1984, et pour l'essentiel en 1987, puisque seuls 48 sièges avaient alors été pourvus au scrutin individuel, contre 400 au scrutin de liste.

28 May Kassem, op.cit.

8 
la justice pour « corruption de la vie politique », laissant désormais le champ libre à la compétition inter-partisane. Dès lors, les partis politiques cherchèrent à s'articuler aux intérêts des électeurs, et contribuèrent à la politisation du vote, et de l'électorat, lors des élections législatives de 2011-2012, d'autant plus que le scrutin de liste avait fait son retour lors de ces élections, en présidant à l'élection de deux tiers des députés, le tiers restant étant élu au scrutin individuel ${ }^{29}$. Les élections présidentielles qui s'ensuivirent, par leur nature même (un seul siège disponible, organisation en deux tours favorisant la polarisation de l'électorat) accentuèrent encore la politisation du corps électoral.

Enfin, la restauration autoritaire amorcée en 2013 s’accompagna elle aussi de modifications du cadre juridique. Si la loi des partis demeura inchangée, la loi électorale de 2015 mit en place un type de scrutin mixte encore inédit, avec quatre cinquièmes des sièges attribués au scrutin individuel, et le reste pourvu au scrutin de liste, mais non proportionnel : la liste remportant la majorité absolue des voix d'une circonscription à l'issue du premier ou du second tour se voyant octroyer la totalité des sièges de ladite circonscription (règle dite du winner-take-all). Mais ce furent surtout les lois sécuritaires qui jouèrent un rôle important dans cette période, en limitant de facto la compétition politique aux forces qui avaient soutenu le renversement des Frères musulmans durant les mobilisations du 30 juin 2013. La loi réprimant les manifestations (24 novembre 2013), puis le renforcement de l'arsenal anti-terroriste permirent de juguler la mobilisation populaire, de déclarer hors-la-loi non seulement les Frères musulmans, mais aussi la principale organisation de la jeunesse révolutionnaire, le Mouvement du 6 Avril, et de priver en pratique de tout moyen d'action les partis politiques ouvertement opposés au régime, pour l'essentiel islamistes (Construction et Développement, Wasat, Watan). Dans ce contexte, l'on n'assista pas durant les élections législatives de 2015 à un retour aux anciennes pratiques clientélistes, et les anciens députés du PND peinèrent à revenir dans le jeu politique, malgré le recours - dans bien des cas - à l'achat de voix ${ }^{30}$. Le corps électoral exprima alors avant tout son allégeance à l'État et son désir d'un retour à l'ordre en élisant de nombreux députés liés à différents corps de la police ou de l'armée, sans trop se préoccuper de leurs étiquettes partisanes. De fait, les députés indépendants sont majoritaires au sein de ce Parlement, dans lequel les partis politiques demeurent faibles et dispersés (le plus important, le Parti des Égyptiens libres, rassemble à peine plus de $10 \%$ des sièges). Dans la même période, les élections présidentielles de 2014, et plus encore celles de 2018, illustrèrent le paradoxe d'une mobilisation sans politisation. Si une partie du corps électoral continue d'exprimer à travers son vote son soutien au régime, ce dernier est contraint de recourir à toujours davantage d'expédients (distribution de denrées au-dessous du prix du marché, pressions sur les fonctionnaires ou les employés de grandes entreprises ${ }^{31}$ ) pour maintenir un taux de participation élevé.

\section{Les élections, à la fois révélatrices des tensions et lieu de recherche d'une solution}

La crise du PND évoquée en première partie de cet article marqua de son empreinte les élections législatives de 2010, et en firent l'une des causes immédiates de la révolution de $2011^{32}$. Cette année-là, pour la première fois, le contrôle des élections législatives échappa en effet à la vieille garde du parti hégémonique. Ce fût Ahmad `Ezz qui, en tant que Secrétaire général aux affaires organisationnelles du parti, orchestra la campagne du PND. Le but des fidèles de Gamâl Moubarak était alors de montrer qu'ils étaient capables de surpasser leurs aînés dans ce qui constituait l'un de leurs derniers domaines réservés : l'organisation des élections. En accordant l'investiture à plusieurs députés par circonscriptions, ils essayèrent de masquer le phénomène des candidatures dissidentes

29 Clément Steuer, «Le printemps des partis ? Le rôle des organisations partisanes égyptiennes dans les élections législatives », Confluences Méditerranée, n 82, 2012, p. 91-105.

30 Mahmoud Abou Kassem, et. al., op. cit.

31 Voir par exemple, Mada Masr [en ligne], 28 mars 2018, https://www.madamasr.com/en/2018/03/28/feature/politics/sugar-rice-and-everything-nice-mobilizing-voter-turnout-inegypts-presidential-election/, consulté le 6 juillet 2018.

32 Sur l’organisation de ces élections, voir Florian Kohstall et Frédéric Vairel, « Introduction. Les élections législatives et présidentielles en Égypte de 2005 à 2010 », Égypte/Monde arabe, 3e série, nº 7, 2010, p. 1-18. 
au sein du PND. Un pas supplémentaire fut ainsi franchi dans l'acceptation par le parti hégémonique d'une concurrence entre les élites locales organisée sous sa houlette, le PND cherchant à maîtriser le phénomène tout en étouffant sa visibilité. Mais conjuguée au retour à des pratiques massives de fraude, l'exacerbation de la concurrence au sein du parti hégémonique entraîna une quasi-éradication des partis d'opposition, Frères musulmans compris. Ce succès s'avéra bientôt à double tranchant, poussant une opposition jusqu'alors plus ou moins loyale (Wafd, Parti du Rassemblement, parti arabe démocratique nassérien, voire dans une certaine mesure les Frères musulmans) à rejoindre dans la rue des contestataires plus radicaux ${ }^{33}$ (Association nationale pour le changement, Mouvement du 6 Avril).

Les élections législatives et présidentielles de 2011-2012, du fait de leur politisation et de l'articulation des partis et candidats sur les clivages sociaux traversant l'électorat, révélèrent et accentuèrent une forte polarisation de la société appuyée sur d'importantes disparités territoriales. En effet, alors que les deux plus grandes villes du pays (Le Caire et Alexandrie) accordaient volontiers leurs suffrages aux révolutionnaires, le Delta soutenait la contre-révolution, tandis que les campagnes de la vallée du Nil votaient massivement pour les Frères musulmans, et que les marges désertiques se tournaient vers les salafistes. Ainsi, alors que le cœur du pays, davantage développé et intégré à l'économie mondiale, était dominé par les forces séculières, les zones laissées-pourcompte avaient provoqué un raz-de-marée islamiste au Parlement et envoyé un Frère musulman, Mohammed Morsi, occuper le palais présidentiel. Ce constat joua un rôle important dans le procès en légitimité instruit contre les islamistes au pouvoir dans la période 2012-2013, en alimentant parmi les populations des villes et du Delta une angoisse de submersion par les masses paysannes du Sud, et de désintégration du pays sous l'effet de forces centrifuges favorisant l'installation de groupes jihadistes armés aux frontières du pays (Nord-Sinaï, mais aussi désert libyque). Même suite à la restauration autoritaire, la carte de l'abstention - notamment durant le référendum constitutionnel et les élections législatives de 2014 - montre que les déséquilibres territoriaux affectant l'Égypte ont toujours un effet politique, les marges et le Sud s'abstenant davantage que le cœur du pays.

Enfin, les élections de 2015 virent s'affronter deux tentatives concurrentes de restaurer le lien entre l'État et la société rompu par la disparition du PND ${ }^{34}$. D'un côté, une partie de l'appareil d'État appuya la création du Parti de l'Avenir de la Patrie, dont la direction était confiée à Mohammed Badran, alors âgé de 24 ans. Perçu comme un protégé du président de la République, le jeune homme avait été l'un des leaders de l'Union des étudiants, au sein de laquelle une partie de la direction et des cadres du parti avaient été recrutés. De l'autre côté, une coalition électorale baptisée «Pour l'amour de l’Égypte » chercha à fédérer partis politiques et «personnalités » indépendantes soutenant le président Sissi. Cette coalition rassemblant la quasi-totalité des partis politiques en compétition - à l'exception des salafistes du parti Nour, des organisations socialistes et libérales les plus "révolutionnaires » et de quelques petits partis - ne disputa que les 120 sièges pourvus au scrutin de liste, et les remporta d'ailleurs tous du fait de la règle du winner-take-all. Les partis membres de cette coalition entrèrent par contre séparément en compétition pour les 448 sièges pourvus au scrutin individuel. Suite aux élections, les leaders de « Pour l'amour de l’Égypte » cherchèrent à la transformer en bloc majoritaire au Parlement, mais les plus importants partis la composant - y compris le Parti de l'Avenir de la Patrie - s'en retirèrent bientôt. Ces deux initiatives, le Parti de l'Avenir de la Patrie et la coalition « Pour l'amour de l'Égypte », avaient en commun de vouloir moderniser la relation État-société, le parti en remplaçant les élites traditionnelles par une nouvelle élite résultant de la fusion de jeunes révolutionnaires ralliés au régime, de représentants de différentes organisations professionnelles, et de technocrates issus des

33 Clément Steuer, « Les partis politiques égyptiens dans la révolution », L’année du Maghreb, vol. 8, 2012, p. 181192.

34 Mada Masr [en ligne], 14 mars 2016, http://www.madamasr.com/en/2016/03/14/feature/politics/anatomy-of-anelection/, consulté le 3 juillet 2018. 
administrations, et la coalition en les noyant dans un ensemble plus vaste dont le noyau dur serait constitué par des partis politiques à l’idéologie plus ou moins définie.

Ce dernier épisode souligne les difficultés rencontrées par le régime pour unifier autour de lui l'hétéroclite « coalition du 30 Juin » qui lui a donné naissance. Ces difficultés n’ont jusqu’à présent pas été surmontées, comme le montre la coexistence, durant les élections présidentielles de mars 2018, de plusieurs organisations menant campagne pour la réélection du président Sissi. Suite à ces élections, le projet de fusionner les partis existants en trois ou quatre grandes organisations aux contours idéologiques clairement définis fut présenté de manière insistante par des personnalités proches du pouvoir dans les médias. Le régime est donc toujours à la recherche d'une formule lui permettant de restaurer le lien avec la société : si les deux dernières décennies du règne de Moubarak avaient été marquées par une organisation des réseaux de clientèle reliant en cascade le centre du pouvoir aux périphéries du pays, la montée de nouvelles élites, la politisation du corps électoral et l'aggravation des déséquilibres territoriaux ont eu raison de ce système. La période 2011-2012 fut marquée par une tentative de le remplacer par un système démocratique, qui donnerait aux partis politiques les moyens de jouer leur rôle de relais de la demande sociale et de sélection des élites politiques. Mais les processus électoraux agirent en catalyseur de la polarisation de la société égyptienne, la divisant en deux camps dont l'affrontement fit voler en éclat le jeune cadre démocratique. Depuis, le régime issu du 30 juin 2013 n'essaye pas faire revivre un système clientéliste depuis longtemps entré en crise, mais cherche une formule de modernisation illibérale des rapports État-société. Les élections parlementaires de 2015 ont montré qu'il n’existait pas de consensus au sein du régime quant à la stratégie à mener, et aucun des deux projets concurrents dans ces élections n'a été en mesure de prouver sa viabilité. D’où l'importance des élections législatives de 2020. À l'heure où nous mettons un point final à cet article, les élections à l'Assemblée consultative (restaurée par la réforme de constitutionnelle de 2019 après avoir été supprimée par la Constitution de 2014) témoignent d'une tentative de fusion des deux précédentes stratégies : une liste nationale unifiée clairement dominée par le Parti de l'Avenir de la Patrie et rassemblant autour de lui les autres partis pro-régime obtint la totalité des 100 sièges pourvus au scrutin de liste. Occupant 70 places sur cette liste, le Parti de l'Avenir de la Patrie remporta donc automatiquement autant d'élus, les 30 places restantes étant réparties entre les autres partis membres de la coalition. Sur les 100 sièges attribués au scrutin individuel, 88 furent également conquis par le Parti de l'Avenir de la Patrie (dont 68 dès le premier tour), 6 par le parti populaire républicain (lui aussi membre de la liste nationale unifiée), et 6 par des candidats indépendants. Ces élections à la Chambre haute peuvent ainsi être perçues comme une sorte de répétition générale pour les élections législatives prévues pour être organisées du 24 octobre au 8 décembre 2020. 